Clin Obstet Gynecol. 2017 September ; 60(3): 477-484. doi:10.1097/GRF.0000000000000291.

\title{
Surgery and Endometriosis
}

\author{
Austin Zanelotti, MD and Alan H. DeCherney, MD+
}

+NICHD/NIH, Bethesda, Maryland *University of Miami/Jackson Memorial Hospital Department of OB/GYN, Miami, Florida

hEndometriosis is a complex disease involving dysregulation of hormone expression, hormone receptor activation and gene activation. Studies have shown that the basis of lesion occurrence involves increased prostaglandins, cytokines, endogenous estradiol and lack of response to progesterone (9). Regardless of how the endometrial deposits were initiated the penetrance of disease in the population is extremely diverse. Due to the varied expression of disease severity, treatment of endometriosis cannot be considered a one size fits all paradigm. Patient chief complaint, presenting symptom, future fertility desires and age must all be considered in designing a treatment regiment for symptom relief. Traditionally patients have been offered conservative medical management such as oral contraceptives (OCP), progestins, gonadotropin releasing hormone analogs (GnRHa), danazol and aromatase inhibitors for first line therapy. However, surgical intervention has always been an adjunct to medical management in the treatment protocol for endometriosis and chronic pelvic pain (CPP). A careful discussion of risks involved with surgical intervention must be had between patient and provider, the balance of symptom relief and complication risk must be weighed. Laparoscopy is the gold standard in both diagnosing and managing endometriotic lesions and implants. Laparoscopy is the mainstay of surgical intervention due to decreased postoperative recovery time, pain and infection rates over laparotomy (2). Laparotomy conversion procedures are still performed in rare cases of difficult visualization with extensive disease and the need for multiple complex procedures involving other organ structures.

Surgical procedures can be considered either conservative or definitive. Conservative therapy is fertility sparring and involves ablation or excision of peritoneal implants, resection of deep infiltrating implants and removal of endometriomas. While definitive surgical treatment is considered hysterectomy with or without oophorectomy, compromising further fertility. With endometriosis effecting 5-10\% of women of reproductive age there is a large population of patients undergoing surgical management (7). Studies have shown that surgical intervention for endometriosis related pelvic pain significantly decreases postoperative pain $(4,5,41)$. Abbott et al has shown that post operative pain scores and quality of life assessments were significantly improved in regards to dysmenorrheal, nonmenstrual pelvic pain, dyspareunia and dyschezia for a period of up to 5 yrs postoperatively. A recent meta-analysis concluded statistically significant benefit of laparoscopic surgery for

Corresponding author: Austin Zanelotti, MD*, University of Miami/Jackson Memorial Hospital Department of OB/GYN 3232, Coral Way \#1307 Miami, FL 33145, austin.zanelotti@jhsmiami.org. 
the effective treatment of pain due to endometriosis in comparison to diagnostic laparoscopy with no treatment (8).

Position and depth of invasion of endometriotic implants greatly impacts the procedure performed. Deeply infiltrating endometriosis (DIE) consists of endometriotic nodules that invade greater than $5 \mathrm{~mm}$ into the peritoneal or organ surface. The type of pelvic pain is correlated to the location of the DIE implants and can aid in pre-operative assessment of each patients individualized symptoms (1). Patients with DIE are more likely to have noncyclic CPP, most likely related to the infiltration of subperitoneal or visceral nerves by the implant. This is facilitated by activation of prostaglandins and chemokines associated with local angiogenic and neurogenic environments. This is thought to increase C-type nerve fibers and increase sensation of CPP due to the constant inflammatory state that endometriosis creates (15). The stroma of these lesions expresses receptors for nerve growth factor (NGF), which aids in recruitment of sensory nerve fibers (16). This increase in innervations allows for further pain perception in affected individuals. The increase in these nociceptors is further enhanced by sensitization with estrogen, which is found in abundance due to local implant estradiol (E2) production. Complete resection or ablation of these lesions is paramount in surgical treatment of endometriosis and more specifically DIE. This can involve resection of peritoneum, uterosacral ligament, posterior vagina, anterior rectum, and intestine (shaving or resection with anastomosis). Other procedures used in combination with traditional ablation and resection techniques are laparoscopic uterine nerve ablation (LUNA) and laparoscopic presacral neurectomy (LPSN). These adjunct procedures were thought to increase overall pain relief. LUNA was designed to disrupt efferent nerve fibers in the uterosacral ligaments to decrease uterine pain associated with dysmenorrhea. LPSN involves interrupting the sympathetic innervations to the uterus at the superior hypogastric plexus. However, after a Cochrane review it was noted that both LUNA and LPSN provided no increase in short term pain relief compared to conventional laparoscopic treatment (40). A large randomized controlled trial comparing results of conservative laparoscopic surgery to laparoscopic surgery with LUNA observed no difference between groups in the percentage of patients having recurrent dysmenorrhea one and three years following surgery (11). LPSN was noted to have some advantage in long-term pain relief in midline abdominal pain only. There is an increased incidence of post-operative constipation and urinary dysfunction due to PSN (14). It also must be considered that LPSN is a more technically challenging procedure with increased risk of bleeding complications due to surrounding venous plexus, requiring a skilled laparoscopic surgeon.

A recent meta-analysis of $10 \mathrm{RCTs}$ showed that Laparoscopic surgery was associated with decreased overall pain (measured as 'pain better or improved') compared with diagnostic laparoscopy, both at six months (odds ratio (OR) 6.58,95\% CI 3.31 to 13.10 ) and at 12 months (OR 10.00, 95\% CI 3.21 to 31.17) (17). Following successful procedures, the issue of disease recurrence or re-exacerbation can be high. In a study of 2-5 yr follow up, 33\% of women required further surgical intervention, women who had more severe disease were more likely to require repeat procedures (5). In a retrospective study over $10 \mathrm{yrs}$ and 486 laparoscopic cases for endometriosis, 51\% of women required repeat procedures. Risk of reoperation was increased with presence of endometriosis on ovary, adhesions in the pouch of Douglas, bowel, fallopian tubes or ovaries (6). Women who presented for surgery at an age 
less than 30 were significantly more likely to have a repeat procedure than woman $>30$.

Repeat procedures were lowest amongst women greater than 44 . The discrepancy in age could be due to the fact that older patients are closer in age to menopause at time of procedure and experience a natural decline in estrogen production alleviating symptoms. This also lends to the idea that more pronounced disease is seen in younger reproductive aged women. In a conflicting study, 163 patients in a private practice setting, with histologically confirmed endometriosis on previous laparoscopy had a re-operation rate of $20 \%$ (41). Of those patients undergoing re-operation, biopsy confirmed diagnosis of endometriosis was only seen in $40.62 \%$ of cases. This decrease in re-operation rate could be due to decreased numbers of advanced stage disease compared to studies done at tertiary care centers. The observance of continued pelvic pain with despite no histologic evidence of disease shows that $\mathrm{CPP}$ can be present without recurrence of disease.

Laparoscopic ablation or resection of endometrial implants must be done with caution and a careful understanding of the anatomical structures involved. Occurrence of associated adhesions often present with endometrial implants that can distort normal anatomy and make visualization challenging. When deciding whether to desiccate or ablate an implant in comparison to laparoscopic resection no good RCT trials have supported one technique over the other. Two small trials have been conducted showing no significant difference in postoperative pain scores at 6-12 months $(23,24)$. A five-year follow up study by Healey et al of ablative versus excisional technique did show significant reduction in deep dyspareunia with excision over ablation at multivariate analysis (25). All other parameters reviewed showed no significance in pain scale rating between the two modalities. Decision to use one technique over the other will come down to the individual surgeons preference and comfort level. Prior to implant removal or desiccation careful review of adjacent anatomic structures must be done. If the implant is overlying ureter or blood vessels concern must be had for depth of thermal spread if using ablation. If excision is used, careful dissection of healthy surrounding tissue must be done in order to excise all of the affected tissue. Endometriotic implants cause underlying fibrosis and distortion of adjacent anatomy.

Ovarian Endometriomas represent a distinct variation of disease presence and require a different approach then lesion/implant removal. Endometriomas consist of menstrual debris encapsulated in ovarian tissue creating a pseudocyst. This cyst like structure does not have a true epithelial lining that is seen in other ovarian true cysts. Multiple theories remain on the development of this pseudocyst formation. The original proposed by Hughesdon in 1957 included the invagination of ovarian cortex following the deposition of an endometriotic implant. Subsequent theories have involved metaplasia of coelomic ovarian epithelium and also deposition of an endometrial implant inside a functional ovarian cyst (19). Endometriomas are present in approximately $17-44 \%$ of patients with endometriosis (18). Ability to diagnose endometriomas when a patient presents with pelvic pain is much improved with ultrasound. Unlike endometrial implants that must be diagnose with direct visualization through laparoscopy, endometriomas can be visualized with transabdominal and transvaginal ultrasound. Guerriero et al showed the sensitivity and specificity to differentiate endometriomas from other ovarian cysts at $83 \%$ and $89 \%$, respectively (20). Based on patient symptom severity and size of endometrioma, the decision of surgical resection is made. Larger endometriomas greater than $5 \mathrm{~cm}$ have a higher risk of ovarian 
torsion. Surgical technique of laparoscopic removal of the endometrioma involves cyst drainage, excision (stripping technique), fulguration or ablation of cyst wall. Drainage alone is no longer a recommended treatment modality due to the high prevalence of recurrence. A study of 100 women undergoing laparoscopic cyst excision versus drainage by Marana et al showed a recurrence rate of $4 \%$ and $84 \%$, respectively (21). Cyst wall excision is preferred to the fenestration and ablation technique due to decreased risk of re-operation with excision. A study has shown re-operation rates of $57.8 \%$ with ablation and $23.5 \%$ with excision (22). Excision also decreases post-operative dysmenorrhea, dyspareunia and noncyclic pelvic pain (12).

Surgical management of DIE lesions can be more tedious and complex than treatment of peritoneal endometriosis. Most cases of DIE involve extensive adhesive disease and fibrosis of underlying tissue at implant sites creating pelvic nodules. This requires adhesiolysis in order to mobilize tubes and ovaries to gain access to nodules for attempt at resection or ablation. Most cases of DIE are posterior in nature and consist of lesions on the uterosacral ligaments, posterior vaginal wall and the anterior rectosigmoid colon (3). The uterosacral ligaments are the most frequent location of posterior involvement and are present in $83 \%$ of cases (3). Should pre-operative assessment uncover bowel involvement causing stenosis or ureteral stricture a multidisciplinary approach must be considered. Collaboration with colorectal surgery and urology for planning of possible bowel resection or ureteral reimplantation and re-anastomosis is prudent. Due to the possibility of these procedures becoming complicated and tedious there is an increased risk of visceral and neural lesions. These cases should be considered carefully before proceeding to surgery. Careful evaluation with transvaginal ultrasound (TVUS) and rectal endoscopic sonography should be done if there is suspicion for bowel endometriosis. In one study TVUS had a sensitivity of $92.6 \%$, specificity of $100 \%$ and a positive predictive value of $100 \%$ in the diagnosis of bowel lesions (26). If gastrointestinal complaints are present a colonoscopy with barium enema may be needed pre-operatively. In cases of severe isolated disease of the uterosacral ligaments, unilateral or bilateral excision can be completed, depending on nodule position. Landmarks of excision should include the ureters, uterine arteries, hypogastric nerves, and the rectosigmoid. Careful dissection must be done so as to not enter rectal mucosa or vaginal cavity. If nodules are present in the recto-vaginal space, the surgeon must be conscience of the middle rectal artery during dissection. Once the nodule is isolated dissection should be done so as to remove the least amount of rectal serosa as possible and leave the majority of the lesion on the posterior vaginal wall. Once the rectum is free the nodule can be dissected off the vaginal wall or in cases of deep infiltration the vaginal tissue can be completely resected and then closed with interrupted suture. Pain free improvement was shown at up to 24 months following surgical intervention compared to expectant management in regards to dysmenorrhea (38.9\% vs $24.5 \%)$, dyspareunia $(72.9 \%$ vs $48.2 \%)$ and dyschezia $(78.1 \%$ vs $57.4 \%$ ) in women with proven recto-vaginal disease (12).

When DIE is seen on rectal or colonic mucosa depth of invasion must be assessed. Patients that have multiple bowel implants, sigmoid involvement, lesions larger than $3 \mathrm{~cm}$ and implants that involve greater than $50 \%$ of the circumference of the bowel wall will benefit greater from a resection with re-anastomosis (42). Also any cases involving bowel stenosis should be resected. Patients that have an isolated implant or rectal involvement may be a 
candidate for a shaving or discoid resection procedure. This more conservative approach allows for less nerve interruption, faster recovery and less risk of post-operative bowel dysfunction. The shaving technique allows for resection of superficial lesions involving the serosa or muscularis of the bowel. Careful removal of the implant is done in a skinning technique to the level of the muscularis and the bowel is then oversewn to repair the defect and protect against fistula formation. If the lesion is less than $3 \mathrm{~cm}$ but beyond the mucosa it can be removed trans anally via a discoid resection with a circular stapler. This avoids segmental bowel resection. When performing segmental resection care must be made to dissect close the bowel serosa to preserve vascular lymphatic vessels and nerve tissue. The staple line should be placed so as to give a margin of $1-2 \mathrm{~cm}$ of healthy tissue beyond the implant site to allow adequate resection of all gross disease.

Combined surgical and medical management of endometriosis implants have been shown to decrease implant recurrence rates and improve pain relief $(28,29)$. A Cochrane review showed a significant improvement in disease recurrence with decreased American Fertility Society (AFS) scores at second look laparoscopy (27). The highest cure rate, as defined by an Endoscopic Endometriosis Classification stage of zero, was noted to be by surgical resection or ablation followed by a 3 month course of GnRHa in a study of 450 women in comparison to surgery or medical management alone (29). Medical treatment in the form of continuous or cyclic OCP and GnRHa is designed to suppress estrogen levels and keep remaining endometriotic implants quiescent post operatively. This hormonal suppression does not allow accelerated re-growth of implants and decreases disease recurrence. Some studies have looked at the possibility of pre-operative hormonal suppression to aid in surgical resection of implants. Meta analysis has shown a reduction in implant size and AFS score noted at time of surgery with pre-operative medical therapy (27). No significant impact has been noted regarding extent of resection, time of surgery needed to clear disease or patient outcomes when using neoadjuvant hormonal therapy. Another option to hormonal suppression outside of GnRHa and OCP treatment is the use of levonorgestrel-releasing intrauterine device (LNG IUD). Studies have shown that in comparison to surgical management alone LNG IUD placement significantly decreased dysmenorrhea and noncyclic pelvic pain $(13,33)$.

Definitive management for endometriosis sequelae is removal of bilateral ovaries. This radical approach to surgical management allows for vastly decreased estrogen levels and reduction of implant size and number. The surgical menopause that ensues following bilateral salpingo-oophorectomy (BSO) can greatly impact a patient's life. Negative side effects of decreased bone density and eliminating future fertility necessitates careful discussion between the patient and provider. Patients that are closer in age to the average onset of menopause, 51 years old, may be more inclined to have radical surgery as they are more likely to have completed child bearing and will have fewer years exposed to a hypoestrogenic state. Patients that have had poor response to previous medical and surgical therapy may opt for radical procedures rather than a repeat excisional or ablative procedure. Some women may opt for ovarian conservation with radical surgery and undergo laparoscopic hysterectomy without BSO. This procedure can decrease dysmenorrhea, pelvic pain and dyspareunia but not to the extent of oophorectomy. Namnoum et al showed that women undergoing hysterectomy and $\mathrm{BSO}, 10 \%$ had recurrent pain and $3.7 \%$ required re- 
operation. While those women that underwent hysterectomy with ovarian preservation, $62 \%$ had recurrent pain and $31 \%$ required re-operation (30).

Post-menopausal hormone therapy should be considered in those women undergoing BSO. Treatment should be initiated directly following procedure. Estrogen support can be given in the form of $0.625 \mathrm{mg}$ of conjugated equine estrogens to alleviate negative impacts of bone loss, vasomotor symptoms and mood changes. Low dose estrogen has minimal impact on disease recurrence and was shown to be as low as $3.5 \%$ following estrogen therapy (31). It has been reported that in patients undergoing ovarian suppression with GnRHa and hormonal add back therapy, recurrence of pain does not begin until an E2 level of 40pg/mL (32). Low dose estrogen replacement will not elevate E2 levels beyond this threshold and minimize pain recurrence.

Surgical management of endometriosis has been used as part of the treatment protocol of infertile women with good results. It has been shown that 35-50\% of infertile women have associated endometriosis (34). Surgical correction of tubal and ovarian disease due to adhesions restores normal anatomy and aids in restoring fertility. A multicenter Canadian study showed a significantly higher spontaneous pregnancy rate in women undergoing laparoscopic ablation of peritoneal implants compared to diagnostic laparoscopy only, $36.6 \%$ versus $21.9 \%$, respectively (35). Centini et al showed that laparoscopic removal of DIE lesions produced an overall pregnancy rate of $60 \% ; 38.5 \%$ spontaneously and $21.4 \%$ by assisted reproductive technology (ART)(37). The reduction in implants is hypothesized to reduce pelvic inflammation and allow for natural conception. Peritoneal fluid becomes altered due to implant production of excess prostaglandins, proteases and cytokines (inflammatory, angiogenic, neurogenic) that disrupts activity of the tubal ampulla and normal spermatic function. Restoration of normal tubal anatomy via adhesiolysis allows for proper tubal patency and oocyte capture by fimbriae. Despite the increase in pregnancy rate from surgical intervention, diagnostic laparoscopies should not be performed on asymptomatic patients with infertility (36). Surgical intervention should only be attempted if the patient has severe symptoms and benefits of pain relief outweigh the risks of surgery. Consequences of expectant management must be considered such as ovarian torsion due to large endometrioma, cyst rupture, progression of implants, growth of endometrioma and advancement of recto-vaginal implants. Endometriomas in particular have been associated with a decline in fertility due to disruption of ovarian stroma. It has been suggested that stretching of the ovarian cortex can lead to a loss of primordial follicles. Also, increased inflammation causes elevated oxidative stress to the adjacent cortical tissue. There is an increase in reactive oxygen species and elevated amounts of free iron in chocolate cysts that can be absorbed by surrounding tissue causing a direct gonadotoxic effect. Inflamed cortical tissue increases oxidative stress causing microvascular injury and reduced vascularization which leads to a decrease in antral follicle count (AFC) (38). Barri et al showed that the pregnancy rate of infertile women with endometriomas status post cystectomy was 54.2\%, women treated with expectant management had a pregnancy rate was $12 \%$ (43). Endometrioma resection increases pregnancy rates while decreasing pelvic pain, risk of ovarian torsion and allows for improved access of ovarian follicles should oocyte retrieval be required. Surgical excision of implants and endometriomas must be done with great care. Meta analysis has shown that surgical resection can have a deleterious effect on post- 
operative AMH (39). Surgery can remove healthy ovarian tissue and decrease follicle count. Electrocautery for hemostasis can cause ovarian cortical inflammation and fibrosis. A skilled surgeon must decide the appropriate means of resection or ablation of endometriotic implants and removal of endometriomas to best preserve the patients future fertility capacity and alleviate the patient's pain symptoms.

\section{Resources}

1. Fauconnier A, Chapron C, Dubuisson JB, Vieira M, Dousset B, Bréart G. Relation between pain symptoms and the anatomic location of deep infiltrating endometriosis. Fertil Steril. 2002; 78(4): 719-726. [PubMed: 12372446]

2. Crosignani PG, Vercellini P, Biffignandi F, Costantini W, Cortesi I, Imparato E. Laparoscopy versus laparotomy in conservative surgical treatment for severe endometriosis. Fertil Steril. 1996 Nov; 66(5):706-11. [PubMed: 8893671]

3. Chapron C, Fauconnier A, Vieira M, et al. Anatomical distribution of deeply infiltrating endometriosis: surgical implications and proposition for a classification. Hum Reprod. 2003; 18:157-161. [PubMed: 12525459]

4. Chopin N, Vieira M, Borghese B, Foulot H, Dousset B, Coste J, Mignon A, Fauconnier A, Chapron C. Operative management of deeply infiltrating endometriosis: results on pelvic pain symptoms according to a surgical classification. J Minim Invasive Gynecol. 2005; 12:106-112. [PubMed: 15904612]

5. Abbott JA, Hawe J, Clayton RD, Garry R. The effects and effectiveness of laparoscopic excision of endometriosis: a prospective study with 2-5 year follow-up. Human Reprod. 2003; 18(9):19221927.

6. Cheong Y, Tay P, Luk F, Gan HC, Li TC, Cooke I. Laparoscopic surgery for endometriosis: how often do we need to re-operate? J Obstet Gynecol. 2008; 28:82-85. [PubMed: 18259906]

7. Giudice, LC., Swiersz, LM., Burney, RO. Endometriosis. In: Jameson, JL., De Groot, LJ., editors. Endocrinology. 6th. NewYork: Elsevier; 2010. p. 2356-70.

8. Jacobson TZ, Duffy JM, Barlow D, Koninckx PR, Garry R. Laparoscopic surgery for pelvic pain associated with endometriosis. Cochrane Database Syst Rev. 2009

9. Bulun SE. Endometriosis. N Engl J Med. 2009; 360:268-79. [PubMed: 19144942]

10. The American Fertility Society. Revised American Fertility Society classification of endometriosis: 1985. Fertil Steril. 1985; 43:351-352. [PubMed: 3979573]

11. Vercellini P, Aimi G, Busacca M, Apolone G, Uglietti A, Crosignani PG. Laparoscopic uterosacral ligament resection for dysmenorrhea associated with endometriosis: results of a randomized, controlled trial. Fertil Steril. 2003; 80:310-9. [PubMed: 12909493]

12. Vercellini P, Giuliana P, De Giorgi O, et al. Reproductive perfomance in infertile women with rectovaginal endometriosis: is surgery worthwhile? Am J Obstet Gynecol. 2006; 195:1303-1310. [PubMed: 16707075]

13. Vercellini P, Frontino G, De Giorgi O, et al. Comparison of a levonorgestrel-releasing intrauterine device versus expectant management after conservative surgery for symptomatic endometriosis: a pilot study. Fertil Steril. 2003; 80:305. [PubMed: 12909492]

14. Zullo F, Palomba S, Zupi E, et al. Long-term effectiveness of presacral neurectomyfor the treatment of severe dysmenorrheal due to endometriosis. J Am Assoc Gynecol Laparosc. 2004; 11:23. [PubMed: 15104826]

15. Tokushige N, Markham R, Russell P, Fraser IS. Nerve fibres in peritoneal endometriosis. Hum Reprod. 2006; 21(11):3001-3007. [PubMed: 16950827]

16. Wang G, Tokushige N, Markham R, Fraser IS. Rich innervation of deep infiltrating endometriosis. Hum. Reprod. 2009; 24:827-834. [PubMed: 19151028]

17. Duffy JM, Arambage K, Correa FJ, Olive D, Farquhar C, Garry R, Barlow DH, Jacobson TZ. Laparoscopic surgery for endometriosis. Cochrane Database Syst Rev. 2014 Apr 3.4:CD011031. 
18. Alborzi S, Keramati P, Younesi M, Samsami A, Dadras N. The impact of laparoscopic cystectomy on ovarian reserve in patients with unilateral and bilateral endometriomas. Fertility and Sterility. 2014; 101(2):427-434. [PubMed: 24269044]

19. Kehan S, Hughes C, Price T, Muasher S. An Update on Surgical versus Expectant Management of Ovarian Endometriomas in Infertile Women. Biomed Research International. Vol 2015, Article 204792.

20. Guerriero S, Mais V, Ajossa S, et al. The role of endovaginal ultrasound in differentiating endometriomas from other ovarian cysts. Clin Exp Obstet Gynecol. 1995; 22(1):20-2. [PubMed: 7736636]

21. Marana R, Caruana P, Muzii L, Catalano GF, Mancuso S. Operative lap- aroscopy for ovarian cysts: Excision vs. aspiration. J Reprod Med. 1996; 41:435-8. [PubMed: 8799921]

22. Saleh A, Tulandi T. Reoperation after laparoscopic treatment of ovarian endometriomas by excision and by fenestration. Fertil Steril. 1999; 72:322-4. [PubMed: 10439004]

23. Wright J, Lotfallah H, Jones K, Lovell D. A randomized trial of excision versus ablation for mild endometriosis. Fert Steril. 2005; 83:1830.

24. Healey M, Ang W, Cheng C. Surgical treatment of endometriosis: a prospective randomized double-blinded trial comparing excision and ablation. Fertil Steril. 2010; 94:2536. [PubMed: 20356588]

25. Healey M, Cheng C, Kaur H. To excise or ablate endometriosis? A prospective randomized doubleblinded trial after 5-year follow-up. J Minim Invasive Gynecol. 2014 Nov-Dec;21(6):999-1004. [PubMed: 24768960]

26. Bazot M, Malzy P, Cortez A, et al. Accuracy of transvaginal sonography and rectal endoscopic sonography in the diagnosis of deep infiltrating endometriosis. Ultrsound Obstet Gynecol. 2007; 30:994-1001.

27. Yap C, Furness S, Farquhar C. Pre and post-operative medical therapy for endometriosis surgery. Cochrane Database Syst Rev. 2004; (3)

28. Seracchioli R, Mabrouk M, Frascà C, Manuzzi L, Savelli L, Venturoli S. Long-term oral contraceptive pills and postoperative pain management after laparoscopic excision of ovarian endometrioma: a randomized controlled trial. Fertil Steril. 2010 Jul; 94(2):464-71. [PubMed: 19442968]

29. Alkatout I, Mettler L, Beteta C, Hedderich J, Jonat W, Schollmeyer T, Salmassi A. Combined Surgical and Hormone Therapy for Endometriosis is the Most Effective Treatment: Prospective, Randomized, Controlled Trial. Journal of Minimally Invasive Gynecology. 2013 Jul-Aug;20(4): 473-81. [PubMed: 23567095]

30. Namnoum AB, Hickman TN, Goodman SB, Gehlbach DL, Rock JA. Incidence of symptom recurrence after hysterectomy for endometriosis. Fertil Steril. 1995 Nov; 64(5):898-902. [PubMed: 7589631]

31. Al Kadri H, Hassan S, Al-Fozan HM, Hajeer A. Hormone Therapy for Endometriosis and Surgical Menopause. Cochrane Database Syst Rev. 2009:CD005997. [PubMed: 19160262]

32. Hornstein M, Yuzpe A, Burry K, Heinrichs L, Buttram V, Orwoll E. Prospective randomized double-blind trial of 3 versus 6 months of nafarelin therapy for endometriosis associated pelvic pain. Fertil Steril. 63(1995):955-962. [PubMed: 7720940]

33. Tanmahasamut $P$, Rattanachaiyanont $M$, Angsuwathana $S$, Techatraisak K, Indhavivadhana S, Leerasiri P. Postoperative levonorgestrel-releasing intrauterine system for pelvic endometriosisrelated pain: a randomized controlled trial. Obstet Gynecol. 2012 Mar; 119(3):519-26. [PubMed: 22314873]

34. Giudice L, Kao L. Endometriosis. The Lancet. 2004; 364(9447):1789-1799.

35. Marcoux S, Maheux R, Berube S. Canadian Collaborative Group on Endometriosis. Laparoscopic surgery in infertile women with minimal or mild endometriosis. N Engl J Med. 1997; 337(4):217222. [PubMed: 9227926]

36. Practice Committee of the American Society for Reproductive Medicine. Endometriosis and infertility: a committee opinion. Fetil Steril. 2012; 98(3):591-598. 
37. Centini G, Afors K, Murtada R, Mate Argay I, Lazzeri L, Akladios C, Zupi E, Petraglia F, Wattiez A. The impact of laparoscopic surgical management of deep endometriosis on pregnancy rate. $\mathrm{J}$ Minim Invasive Gynecol. 2015 Sep 28.

38. Kitajima M, Dolmans M, Donnez O, Masuzaki H, Soares M, Donnez J. Enhanced follicular recruitment and atresia in cortex derived from ovaries with endometriomas. Fertil Steril. 2014; 101(4):1031-1037. [PubMed: 24502890]

39. Raffi F, Metwally M, Amer S. The impact of excision of ovarian endometrioma on ovarian reserve: a systematic review and meta-analysis. Journal of Clinical Endocrinology and Metabolism. 2012; 97(9):3146-3154. [PubMed: 22723324]

40. Proctor ML, Latthe PM, Farquhar CM, Khan KS, Johnson NP. Surgical interruption of pelvic nerve pathways for primary and secondary dysmenorrhoea. Cochrane Database Syst Rev. 2005 Oct 19. (4)

41. Roman H, Quibel S, Auber M, Muszynski H, Huet E, Marpeau L, Tuech JJ. Recurrences and fertility after endometrioma ablation in women with and without colorectal endometriosis: a prospective cohort study. Hum Reprod. 2015 Mar; 30(3):558-68. [PubMed: 25574030]

42. Wattiez A, Puga M, Albornoz J, Faller E. Surgical strategy in endometriosis. Best Pract Res Clin Obstet Gynaecol. 2013 Jun; 27(3):381-92. [PubMed: 23340291]

43. Barri PN, Coroleu B, Tur R, Barri-Soldevila PN, Rodríguez I. Endometriosis-associated infertility: surgery and IVF, a comprehensive therapeutic approach. Reprod Biomed Online. 2010 Aug; 21(2): 179-85. [PubMed: 20541976] 